\title{
INFERENCE OF S-SYSTEM MODELS OF GENE REGULATORY NETWORKS USING IMMUNE ALGORITHM
}

\author{
TOMOYOSHI NAKAYAMA, SHIGETO SENO, YOICHI TAKENAKA \\ and HIDEO MATSUDA* \\ Department of Bioinformatic Engineering \\ Graduate School of Information Science and Technology \\ Osaka University, 1-5, Yamadaoka, Suita, Osaka, 565-0871, Japan \\ *matsuda@ist.osaka-u.ac.jp
}

Received 25 July 2011

Revised 5 September 2011

Accepted 15 September 2011

\begin{abstract}
The S-system model is one of the nonlinear differential equation models of gene regulatory networks, and it can describe various dynamics of the relationships among genes. If we successfully infer rigorous S-system model parameters that describe a target gene regulatory network, we can simulate gene expressions mathematically. However, the problem of finding an optimal S-system model parameter is too complex to be solved analytically. Thus, some heuristic search methods that offer approximate solutions are needed for reducing the computational time. In previous studies, several heuristic search methods such as Genetic Algorithms (GAs) have been applied to the parameter search of the S-system model. However, they have not achieved enough estimation accuracy. One of the conceivable reasons is that the mechanisms to escape local optima. We applied an Immune Algorithm (IA) to search for the S-system parameters. IA is also a heuristic search method, which is inspired by the biological mechanism of acquired immunity. Compared to GA, IA is able to search large solution space, thereby avoiding local optima, and have multiple candidates of the solutions. These features work well for searching the S-system model. Actually, our algorithm showed higher performance than GA for both simulation and real data analyses.
\end{abstract}

Keywords: S-system; immune algorithm; gene regulatory network; systems biology.

\section{Introduction}

Recent advancements in gene expression profiling technologies allow us to use massive amounts of gene expression data. We can use these data for revealing complex biological systems, such as gene regulatory networks (GRNs). The inference of GRNs is to reconstruct the GRNs as a mathematical model. The inferred model from detailed data makes the interactions among genes clearly visible and it helps biologists make hypotheses, design their experiments, and reveal the mutual relationships. 
Numerous GRN models have been proposed. The models range from abstract Boolean networks ${ }^{1}$ to more detailed differential equation-based models ${ }^{2,3}$ e.g. the S-system model. ${ }^{4}$ The former model simply approximates a gene expression level as a logical function, which means that a gene is expressed or not expressed. However, real gene expression data show that gene expression levels tend to be continuous rather than binary. The latter model has a structure that possesses physical meanings such as the amount of activation or degradation and can capture the dynamics of the gene expression.

The S-system model ${ }^{5}$ is based on a set of nonlinear ordinary differential equations of the form:

$$
\frac{d x_{i}}{d t}=\alpha \sum_{j=1}^{N_{g}} X_{j}^{g_{i j}}-\beta \sum_{j=1}^{N_{g}} X_{j}^{h_{i j}}
$$

where $N_{g}$ is the number of genes in the GRN and $X_{i}$ is the expression level of gene $i ; \alpha$ and $\beta$ are the rate constants of activation and degradation of gene $i$, respectively. A positive value of $g_{i j}$ indicates that gene $j$ activates gene $i$. A negative value indicates that gene $j$ inhibits the activation of gene $i$. Similarly, a positive value of $h_{i j}$ indicates that gene $j$ increases degradation of gene $i$, and a negative value represents that gene $j$ inhibits the degradation of gene $i$. And the zero values of $g_{i j}$ and $h_{i j}$ mean gene $j$ and gene $i$ have no relations. The total number of these parameters is $2 N_{g}\left(N_{g}+1\right)$. When these S-system parameters are decided, we can simulate the dynamics of the gene expressions by solving the differential equations.

To reconstruct GRNs from gene expression profiles based on the S-system model, an optimization problem must be solved for estimating its parameters. However, it is not easy to find the best solution because of the high complexity of the formula that has a large solution space and existence of many local optima that are similar to each other. This problem is called "inverse problem," and many approaches have been developed to solve it. ${ }^{1}$

While analytical approaches are almost impracticable for solving this problem, evolutionary approaches, such as Genetic Algorithm (GA), ${ }^{6}$ is more practicable. ${ }^{7-10}$ GA can search the large solution space, avoiding local optima. However, inference of the S-system model parameters by the classical GA is too difficult to optimize with the high accuracy. One of the conceivable reasons is that the mechanisms to escape local optima when the search converges into the local optima are not enough.

In this paper, we propose an algorithm that finds the more suitable Ssystem parameters for GRNs using Immune Algorithm (IA). ${ }^{11}$ IA improves GA to cope with the problem to enhance the mechanisms to avoid converging into the local optima. The effectiveness of IA for a complex optimization problem has been studied. ${ }^{12,13}$ By applying IA to the inference problems of the S-system model that formulates a GRN, one can obtain higher accurate performance than GA. 


\section{Method}

\subsection{Basic problem definition}

The problem of inferring GRNs using the S-system model with time-series data is generally formulated as a function optimization problem to minimize the following sum of the squared relative error.

$$
f_{v}=\sum_{i=1}^{N_{g}} \sum_{t=1}^{T}\left(\frac{X_{C, i, t}-X_{I, i, t}}{X_{I, i, t}}\right)^{2}
$$

where $f_{v}$ shows the fitness of an antibody $v ; X_{C, i, t}$ is a $i$ th gene expression level at time point $t$ from the time-course data calculated from the S-system parameters of $v ; X_{I, i, t}$ is a $i$ th gene expression level at time point $t$ from the input data; $I$ means "Input data," and $T$ is the number of time points of input data. Here, we use the fourth-order Runge-Kutta method to calculate the time-series data.

\subsection{Immune Algorithm for S-system model}

Immune Algorithm (IA) ${ }^{11}$ is a heuristic search algorithm that mimics the simple mechanism of acquired immunity. In the immune system, the antibodies are produced to bind the antigen. At that time, the immune system produces diverse antibodies by recognizing the strength of the binding of an antibody to its antigen is called its affinity between antigens and antibodies or between antibodies to acquire more effective antibodies to the antigen. If effective antibodies are produced, the immune system memorizes them as memory-cells to response quickly to the same antigen and as suppressor-cells to suppress immune responses of other cells.

IA enhances the search ability by introducing the memory mechanisms into GA. The characteristics are high diversity and self-control mechanism. It is expected that optimal solutions can be obtained more effectively than by GA. Here, conceptual flowchart of IA is shown in Fig. 1.

IA assumes a target problem as an antigen and its solutions as antibodies, and searches for the antibody that has the highest affinity for the antigen. In inferring GRNs with the S-system model, the antigens are defined as the problem of minimizing the objective function shown in Eq. (2), and each antibody has a candidate set of the S-system model parameters. The goal is to find the antibody that has the highest affinity with the antigen, which means the set of the parameter that minimize Eq. (2). In Step 5, similarity represents how similar an antibody and another are, and concentration represents the number of antibodies that have high similarity. These states of antibodies are used to accelerate or suppress the production of antibodies by memorizing the important antibodies for maintaining high diversity and controlling itself. We show an algorithm based on IA for inferring S-system parameters in detail. 


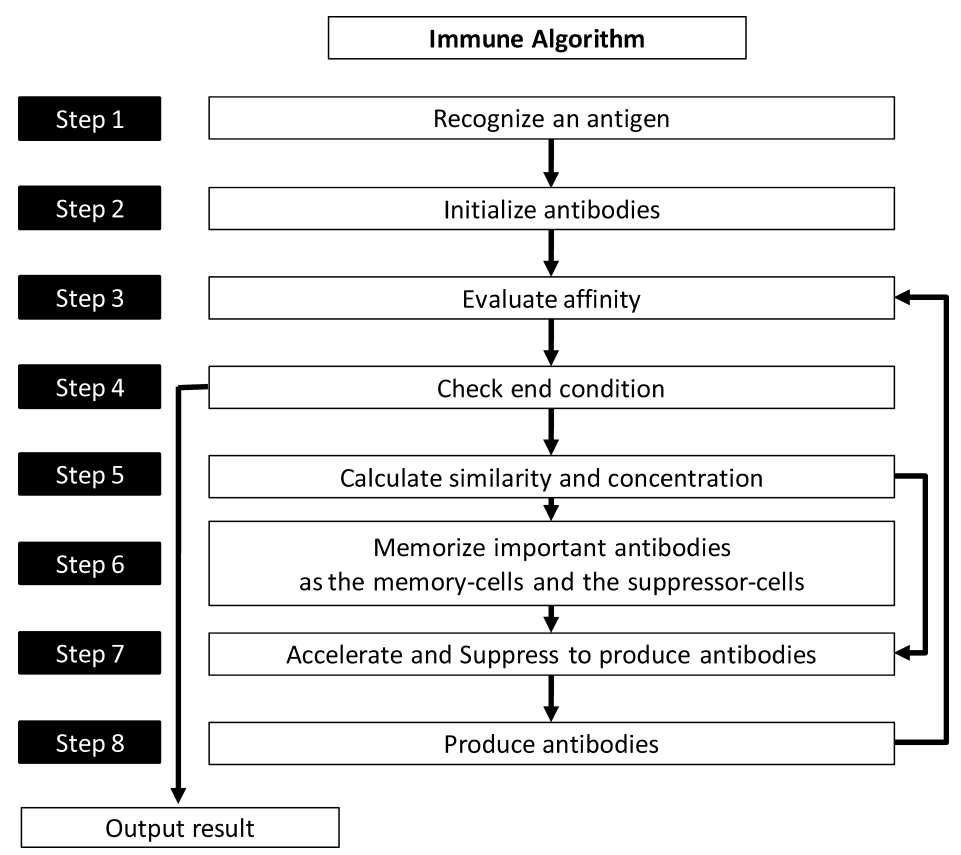

Fig. 1. The flowchart of the proposed method.

\subsubsection{Recognize an antigen}

This recognition step defines the problem to solve as an antigen. In inferring GRN with the S-system model, the antigen is the problem of minimizing the objective function shown in Eq. (2).

\subsubsection{Initialize antibodies}

In this step, initial antibodies of which number is $N_{\gamma}$ are generated. $N_{\gamma}$ is defined as populations. Each antibody is a candidate set of S-system parameters composed of $2 N_{g}^{2}+2 N_{g}$ real numbers that are assigned to randomly in the range of a certain level. Here $N_{g}$ is the number of genes.

\subsubsection{Evaluate affinity}

Evaluate the affinity of antibodies for the antigen. All $N_{\gamma}$ antibodies are evaluated by computing an error between the original time-series data (input data) and the computed time-series data reconstructed from the S-system model with the set of parameters. Affinity $\phi_{v}$ is calculated by the following equation:

$$
\Phi_{v}=\frac{1}{1+f_{v}},
$$

where $f_{v}$ is the fitness that is calculated by Eq. (2). 


\subsubsection{Check end condition}

If this method meets the end condition, IA outputs the memory-cells and terminates. Since IA does not guarantee the convergence to the optimum solution within a fixed number of iterations, we set the number of generations as the end condition, which are the upper limit numbers of the iterations. This is able to compare the performances of GA and IA if the calculation time per generation is equivalent. Section 2.3 explains how to determine the upper limits.

\subsubsection{Calculate similarity and concentration}

This step calculates similarities and concentrations of the antibodies. High concentration of the antibodies indicates that there are many antibodies that have similar parameters in the generation. The concentration is defined as the rate of the number of antibodies whose similarity to the antibody among all antibodies exceeds a concentration threshold. The similarity $\Psi_{v, \omega}$ between antibody $v$ and antibody $\omega$ is calculated by the following equation:

$$
\Psi_{v, \omega}=\frac{1}{1+d(v, \omega)},
$$

where $d(v, \omega)$ is the Euclidean distance between $v$ and $\omega$; and $\Psi_{v, \omega}$ is in the range $\left[1 / N_{\gamma}, 1\right]$. From this, the concentration $\Theta_{v}$ of antibody $v$ is calculated by the following equation:

$$
\begin{gathered}
\Theta_{v}=\frac{1}{N_{\gamma}} \sum_{\omega=1}^{N_{\gamma}} u_{T_{\theta}}\left(\Psi_{v, \omega}\right), \\
u_{T_{\theta}}(t)=\left\{\begin{array}{l}
1\left(t \geq T_{\theta}\right) \\
0\left(t<T_{\theta}\right)
\end{array} .\right.
\end{gathered}
$$

Here, $T_{\theta}$ is the concentration threshold to determine whether the antibodies are similar. The methods of the determination of the thresholds, which is including the other thresholds that appear below, are described in Sec. 2.3.

\subsubsection{Memorize important antibody as the memory-cell and the suppressor-cell}

In this step, the antibodies that have high concentrations are changed as memorycell candidates to create memory-cells, which are the solutions of this algorithm. The memory-cell candidates should have similar parameters to each other, and it is expected as those that one candidate is the local optimum solution or those that there is the local optimum solution in the near distance from their parameters. IA tries a local search to acquire more effective memory-cells to the antigen. In addition, the antibodies are memorized as suppressor-cells to suppress antibodies.

An antibody that has the best affinity among the antibodies of which concentrations exceed memory-cell differentiation threshold $T_{\mu}$ becomes memory-cell 
candidate $\mu^{\prime}$. After that, the best memory-cell candidate $\mu$ is generated from $\mu^{\prime}$ by hill climbing, which is a local search algorithm; $\mu$ becomes a memory-cell and IA memorizes it if the number of memory-cells is less than $M$, which is the limit of memory-cell. If the number of memory-cells is already more than or equal to $M$, a memorized memory-cell that is most similar to $\mu$ is replaced by $\mu$ unless the affinity of the memory-cell is higher than $\mu$.

At the same time, all the antibodies, whose concentrations exceed $T_{\mu}$, are memorized as suppressor-cells $s_{k}\left(k=1, \ldots, N_{s}\right)$. If an antibody that has similarity $\Psi_{v, s k}\left(k=1, \ldots, N_{s}\right)$ exceeds suppressor-cell similarity threshold $T_{s}$, it is replaced a new initialized antibody.

\subsubsection{Accelerate and suppress to produce antibodies}

This step changes the antibodies to produce new effective ones. The effective parameters of antibodies that have high affinity and low concentration become easy to transmit to the next generation. Additionally, the suppressor-cells suppress the production of non-effective antibodies that are similar to the suppressor-cells.

For creating effective antibodies, this step uses a higher half of antibodies, and the lower half of antibodies is replaced by the child generation that is created next step. The rate of antibody $v_{i}$ is calculated as probability $P_{i}$ by the following equations:

$$
\begin{aligned}
P_{i} & =\frac{\varepsilon_{v_{i}}}{\sum_{j}^{N_{\gamma} / 2} \varepsilon_{v_{j}}}, \\
\varepsilon_{v_{i}} & =\frac{\Phi_{v_{i}}}{\sum_{j}^{N_{\gamma} / 2} \Phi_{v_{j}}} \frac{1}{\Theta_{v_{i}}} \prod_{j}^{N_{h}}\left\{1-\Psi_{v_{i}, s_{j}} u_{T_{e}}\left(\Psi_{v_{i}, s_{j}}\right)\right\} .
\end{aligned}
$$

Here, $\varepsilon_{v}$ is an expectation for the performance of antibody $v_{i}$. Expectation of an antibody becomes high when it has high affinity $\Phi_{v i}$ and low concentration $\Theta_{v i}$. If suppressor-cells exist, the antibody $v_{i}$ whose similarity $\Psi_{v i, s}$ exceeds expectation suppressor threshold $T_{\varepsilon}$ becomes low expectation. After that, the suppressor-cells are cleared.

\subsubsection{Produce antibodies}

This step creates an offspring generation by the simplex crossover method SPX. ${ }^{14}$ The method chooses $v_{i}$ for the parents of crossover by probability $P_{i}$, and repeats until the number of chosen $v$ becomes $2 N_{g}\left(N_{g}+1\right)$ for parents without overlap, and their children are formed within the simplex that the parents create.

After the crossover, the parameters of the children mutate with a certain mutation rate $p_{m}$. If the mutation occurs at coefficient $\alpha$ or $\beta$, the values are changed randomly. If it occurs at coefficient $g$ or $h$, the values are changed into zero. This mutation expresses that GRNs are sparse. After mutation, the lower $N_{\gamma} / 2$ antibodies are replaced by the children in descending order of their affinities. 


\subsection{Determination of thresholds and end condition}

Before the inferences, we have to decide the four thresholds; the end condition, which is the number of iterations; and other conditions, because the suitable conditions depend on the input data set. The other conditions are the range of the S-system parameters, the step size of the parameters, the population number, the number of times of local search, and mutation rate $p_{m}$. In this paper, we repeated preliminary experiments to decide in using the input data from which GRNs are inferred.

First, we decided the ranges of the S-system parameters and the other conditions. The lower limit of $\alpha$ and $\beta$ is zero, which means the genes have no relationships, but the upper limit depends on the maximum gene expression level of the data. And the widest range of $g$ and $h$ is $[-3,3]$ in the problem of the GRNs. We have to obtain the upper limit of the range of $\alpha$ and $\beta$, the range of $g$ and $h$, the step size of the parameters, the number of populations, the number of times of local search, and mutation rate $p_{m}$. These conditions are a trade-off between the calculation time and the affinity. We repeated the experiments with a different set of conditions, and we choose the conditions for which the experiment shows wellbalanced performances. In the experiments, the thresholds and the end condition not to occur the replacements of the antibodies are: $\mathrm{T}_{\Theta}=1, T_{\mu}=1, T_{s}=1, T_{\varepsilon}=1$, and the number of generations $=100$.

Then, we performed experiments to decide the thresholds. The range of each threshold is $[0,1]$. The experiments are set with different thresholds at 0.1 intervals, and the total number of the experiments is 1,000 . We repeated the experiments three times and chose the thresholds for which the result of experiments had the best affinity. Next, we repeat the experiments once again. The range of each threshold is from threshold minus 0.05 to threshold plus 0.05 . Likewise, the experiments are performed with different thresholds at 0.01 intervals in 1,000 experiments, with each experiment repeated thrice.

Additionally, we did another preliminary experiment that aims to examine the convergence performances and the calculation time of the algorithms. In this paper, the affinities of GA began to show little change when the generation closed in on 100,000 . The calculation time of GA per generation is similar to that of IA, if IA does not memorize antibodies at the time. So the performances of the algorithms from the preliminary experiments can be compared to decide the number of conditions as the end condition.

\section{Results}

We performed two experiments to evaluate the effectiveness of our method by comparing with GA. An artificial gene expression profile is used in the former experiment, and real gene expression profiles are used in the latter. The results from the artificial data show the performance of the methods from the data without disturbances. 
In the inference of the parameters of GA and IA from the artificial data, the conditions that we set from preliminary experiments are below: range of $\alpha$ and $\beta=$ $[0.00,15.00]$; range of $g$ and $h=[-3.00,3.00]$; step size of $\alpha, \beta, g$, and $h=0.01$; the number of generations $=100,000$; the number of population $=200$; concentration threshold $T_{\Theta}=0.35$; memory-cell differentiation threshold $T_{\mu}=0.7$; suppressorcell similarity threshold $T_{s}=0.3$; expectation suppressor threshold $T_{\varepsilon}=0.3$; the number of times of local search $=20$; and mutation rate $p_{m}=0.005$. The four thresholds are only for IA. When we infer the networks from the real data, the range of $\alpha$ and $\beta$ is changed to [0.00,30.00]. The computer environment we used was a PC cluster that consists of 24 CPUs, which are Xeon X7542 $2.67 \mathrm{GHz}$, and $32 \mathrm{~GB}$ memory in total. Our algorithms were implemented in $\mathrm{C}++$.

\subsection{Artificial data}

We used artificial data that had 5 genes and 30 time points. The artificial data were generated from the S-system parameters, which are obtained from the work done in Ref. 7. The dynamics of the genes is shown in Fig. 2.

In estimation with the artificial data, the execution times are approximately the same between GA and IA (47,480 and 46,736 sec, respectively). The distribution of the affinities of GA and IA are shown in Fig. 3. In Fig. 3, $X$-axis shows the affinity of the experiments that are repeated 10 times in each condition, and $Y$-axis shows the frequency of the affinity. The result of IA is that memory-cells have the best affinity in the experiments. There are significant differences of the distributions

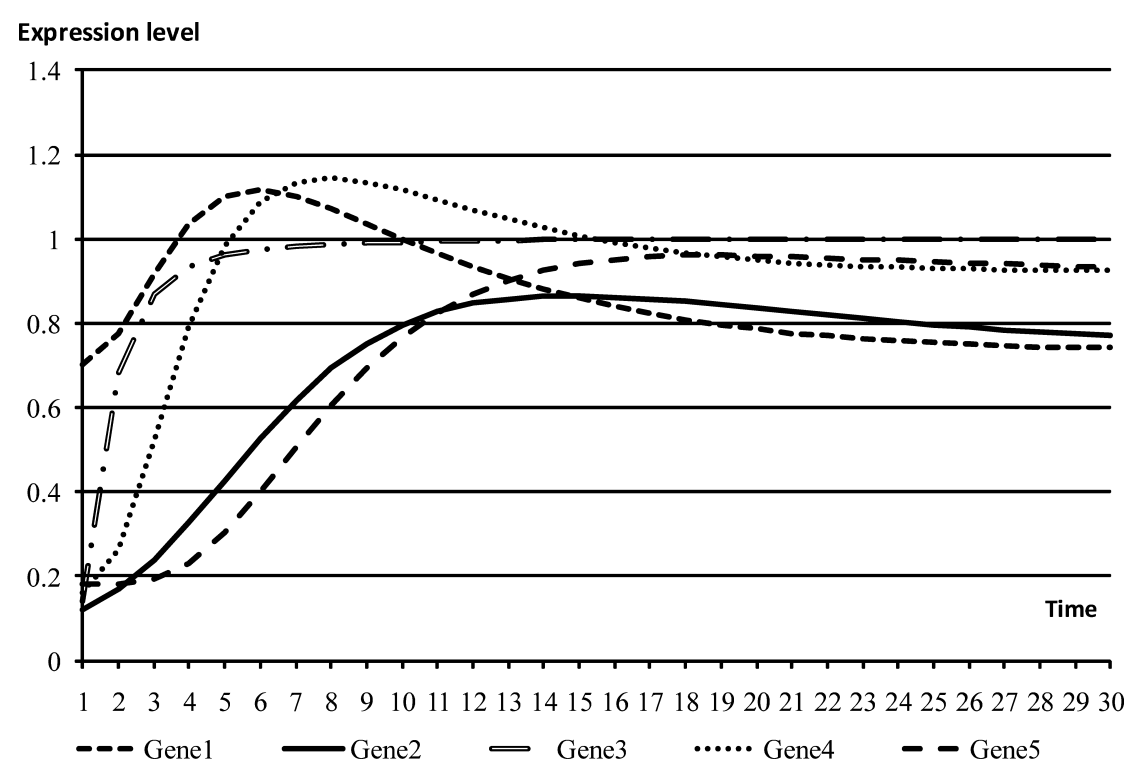

Fig. 2. The time-course expression profiles of the artificial data. 


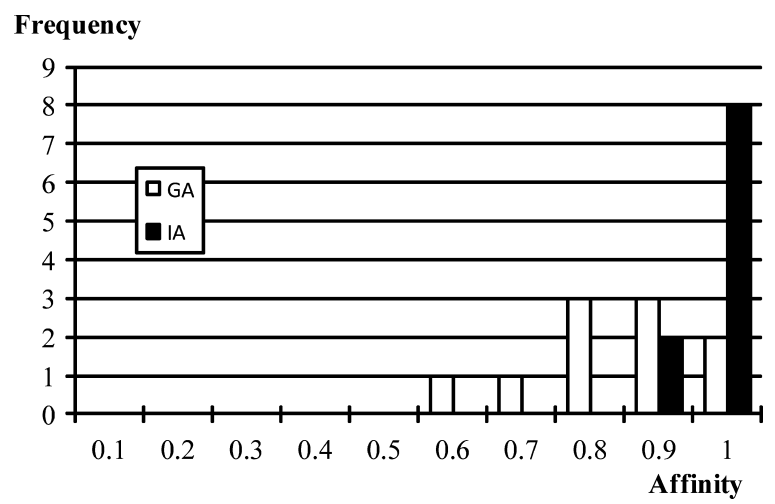

Fig. 3. Histogram of the affinities of inferences for artificial data.

between GA and IA (in Wilcoxon-Mann-Whitney test, $p<0.00013$ ). The standard deviation (SD) of the affinities in GA is 0.165 and that for IA is 0.0363 .

\subsection{Gene expression data}

In this study, we use two experimental time series of the gene expression levels. The dataset 1 consists of 5 genes with 31 time points in adipocyte differentiations of Mus musculus (shown in Fig. 4). The experimental data of time-course expressions of Cebpb, Cebpg, Cebpa, Pparg, and Cebpd were measured with Affymetrix GeneChip

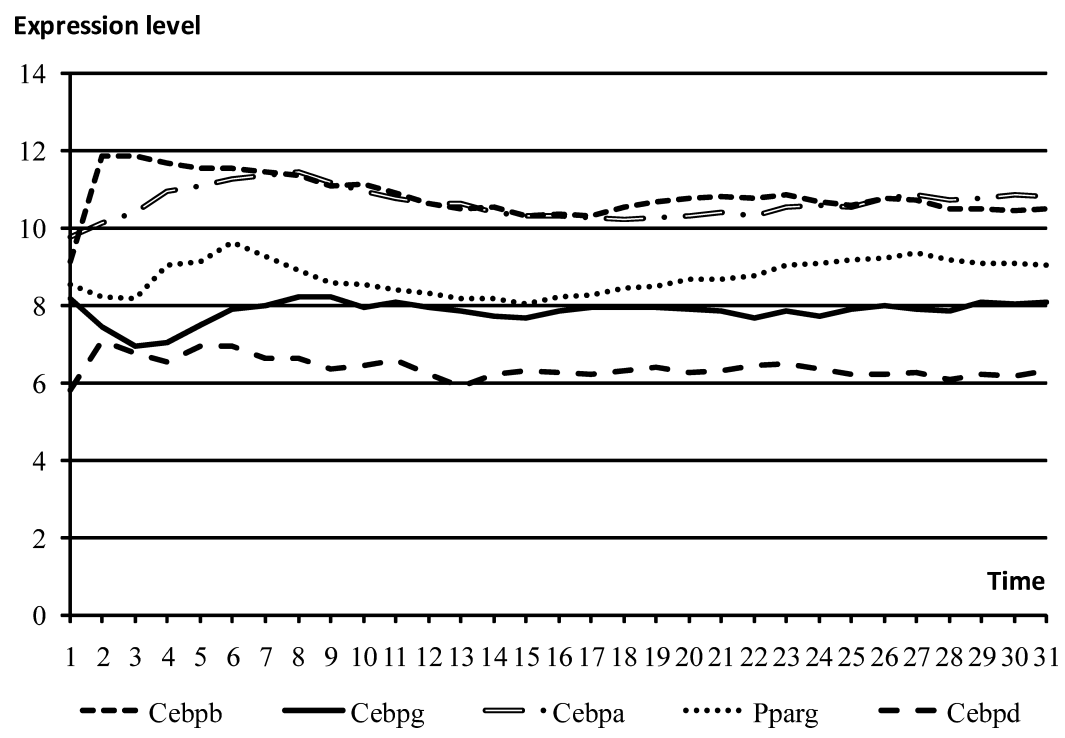

Fig. 4. The time-course expression profiles of the real data (adipocyte). 


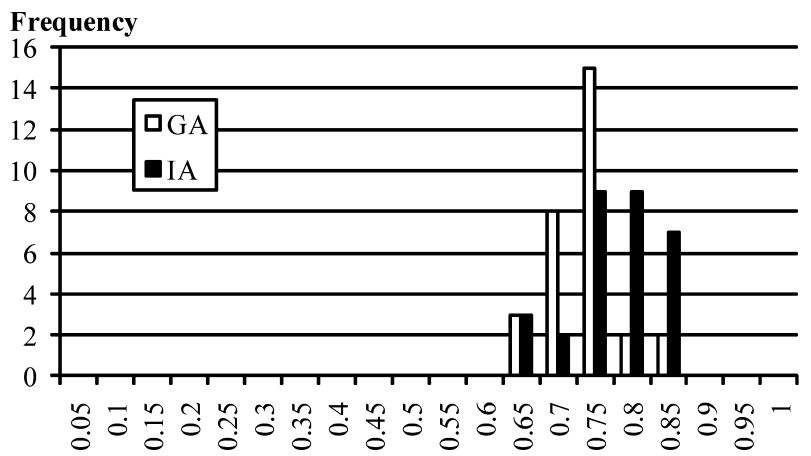

Affinity

Fig. 5. Histogram of the affinities of inferences for real data (adipocyte).

Mouse Genome 430 2.0. The real data contain the genes that are considered to have functional relationships to each other.

In estimation with the real data, IA has longer execution times than GA $(122,807$ and $143,843 \mathrm{sec}$, respectively). The distribution of the affinities of GA and IA is shown in Fig. 5. The $X$-axis shows the affinities of experiments that are repeated 30 times, and the $Y$-axis shows the frequency of the experiments. The results of IA are that memory-cells have the best affinity in the experiments. IA shows better performance than GA (In Wilcoxon-Mann-Whitney test, $p<0.0021$ ), and the SDs of the affinities in GA and IA are 0.0411 and 0.0573 , respectively.

\section{Discussions}

The result shows that IA is more effective to improve the estimation accuracy of GRNs than GA. Most of the affinities of the estimation results by IA achieved higher affinities than GA. Their SDs at the artificial data are smaller than that of GA. It means that the performance of estimations by IA is high and stable. So, it is important and effective to escape from converging into local optima in the inference problem of S-system models.

Meanwhile, we realized that the condition setting of IA takes more time than GA. IA needs four initial conditions of the thresholds in addition to GA, and they seem to affect the performance of IA critically. For example, the higher the concentration threshold $T_{\Theta}$, the lower the concentrations in immune cells. It means that the frequency of differentiation to memory-cells decreases, while the locality of the search increases. In this paper, we repeated small preliminary experiments to obtain the effective thresholds. The thresholds from the method achieved good performances, and the method is applicable to the other data sets. However, it might be better to use another method would be needed to estimate the ranges of the conditions in gene expression data more quickly. 


\section{References}

1. Akutsu T, Miyano S, Kuhara S, Algorithms for identifying Boolean networks and related biological networks based on matrix multiplication and fingerprint function, J Comput Biol 7:331-343, 2000.

2. Hirose O, Yoshida R, Imoto S, Yamaguchi R, Higuchi T, Charnock-Jones DS, Print C, Miyano S, Statistical inference of transcriptional module-based gene networks from time course gene expression profiles by using state space models, Bioinformatics 24(7):932-942, 2008.

3. Mangan S, Alon U, Structure and function of the feed-forward loop network motif, Proc Natl Acad Sci USA 100(21):11980-11985, 2003.

4. Maki Y, Tominaga D, Okamoto M, Watanabe S, EguchiY, Development of a system for the inference of large scale genetic networks, Pac Symp Biocomput 446-458, 2001.

5. Savageau MA, Biochemical Systems Analysis: Study of Function and Design in Molecular Biology, Addison-Wesley Educational Publishers Inc., 1976.

6. Goldberg DE, Genetic Algorithm, Search Optimization and Machine Learning, Addison Wesley, 1989.

7. Kikuchi S, Tominaga D, Arita M, Takahashi K, Tomita M, Dynamic modeling of genetic networks using genetic algorithm and S-system, Bioinformatics 19(5):643650, 2003.

8. Noman N, Iba H, Inference of gene regulatory networks using S-system and differential evolution, GECCO '05 Proc 2005 Conference on Genetic and Evolutionary Computation, 2005.

9. Kimura S, Ide K, Kashihara A, Kano M, Hatakeyama M, Masui R, Nakagawa N, Yokoyama S, Kuramitsu S, Konagaya A, Inference of S-system models of genetic networks using a cooperative coevolutionary algorithm, Bioinformatics 21(7):11541163, 2005.

10. Orland RG, Christoph K, Kirsten J, Prospero CN, Parameter estimation using simulated annealing for S-system models of biochemical networks, 4(23):480-486, 2007.

11. Mori K, Tsukiyama M, Fukuda T, Application of an immune algorithm to multioptimization problems, T. IEE Japan 117-C(5):593-598, 1997.

12. Endoh S, Toma N, Yamada K, Immune algorithm for n-TSP, IEEE International Conference on Systems, Man, and Cybernetics 3844-3849, 1998.

13. Jang-Sung C, Hyun-Kyo J, Song-Yop H, A study on comparison of optimization performances between immune algorithm and other heuristic algorithms, IEEE Transactions on Magnetics 34(5), 1998.

14. Tsutsui S, Yamamura M, Higuchi T, Multi-parent recombination with simplex crossover in real-coded genetic algorithms, Proc. GECCO-99 657-664, 1999.

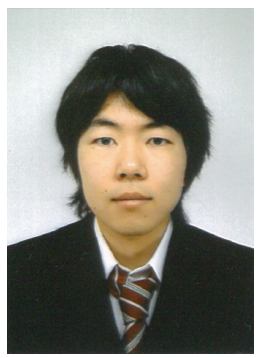

Tomoyoshi Nakayama received his Master's degree in Information Science from Osaka University, Japan, in 2011. He is currently a doctoral candidate at the Department of Bioinformatic Engineering, Graduate School of Information Science and Technology, Osaka University, Japan. 


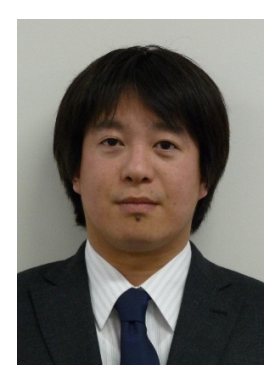

Shigeto Seno received his B.Eng., M.Eng., and Ph.D. degrees in Computer Science from Osaka University, Japan, in 2001, 2003 and 2006, respectively. He is an Assistant Professor in the Department of Bioinformatic Engineering, Graduate School of Information Science and Technology, Osaka University, Japan, since 2006. His research interests include gene expression analysis and statistical learning in bioinformatics.

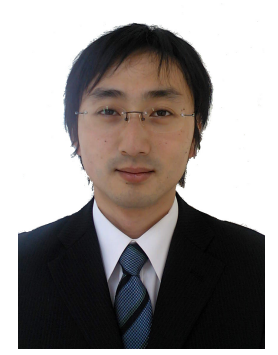

Yoichi Takenaka received his M.Eng. and Ph.D. degrees in Computer Science from Osaka University, Japan, in 1997 and 2000, respectively. He was an Assistant Professor from 2000 to 2002 in Osaka University. Since 2002, he has been an Associate Professor in the Department of Bioinformatic Engineering, Graduate School of Information Science and Technology, Osaka University. His research interests include gene expression analysis, high-throughput sequence analysis, and legal informatics.

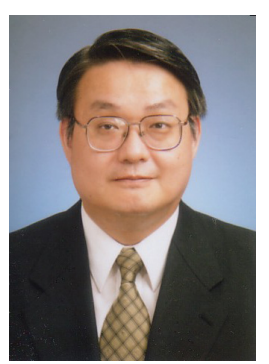

Hideo Matsuda received his B.Sc. degree in Physics from Kobe University, Japan, in 1982, and his M.Eng. and Ph.D. degrees in Computer Science from Kobe University, Japan, in 1984 and 1987, respectively. He was a Research Associate in Kobe University from 1987 to 1990, a Lecturer in Kobe University from 1990 to 1994, an Associate Professor in Osaka University, Japan, from 1994 to 2002. Since 2002, he has been a Professor in the Department of Bioinformatic Engineering, Graduate School of Information Science and Technology, Osaka University. His research interests include genomic information analysis, integrated biological databases, and gene expression analysis. 